\title{
Amount of Rotation About a Point and the Morse Index *
}

\author{
John N. Mather \\ Department of Mathematics, Princeton University, Princeton, NJ 08544, USA
}

\begin{abstract}
For the case of exact, area preserving, monotone twist diffeomorphisms, we give formulas relating the amount of rotation about an orbit and certain Morse indices.
\end{abstract}

\section{Introduction}

This paper continues the study of exact, area preserving, monotone twist diffeomorphisms of the annulus which I have pursued in [14-19]. I should point out that Aubry earlier found ideas similar to many of those in [14-19] (see [1]). See [3] and the references therein for later developments in Aubry's theory. See also Katok $[11,12]$ for other recent developments.

Precise definitions of the terminology used in this introduction will be given in Sects. $2-4$.

An exact, area preserving, monotone twist diffeomorphism $f$ (Sect. 2) admits a definition in terms of a global generating function. It is then possible to give a variational formulation (Sect. 3) of periodic orbits: periodic orbits of type $(p, q)$ are in one-one correspondence with critical points of an "energy" functional $W^{q}$ defined on the space $\mathscr{X}_{p q}$ of "states" of type $(p, q)$. Our first result relates the amount of rotation $\varrho$ about a periodic orbit of type $(p, q)$ and the Morse index $I$ of $W^{q}$ at the corresponding critical point $x$ in $\mathscr{X}_{p q}$.

In order to state this result, we now give the definition of $\varrho$ for the case of a $C^{1}$ diffeomorphism $f$ of $T \times \mathbb{R}=(\mathbb{R} / \mathbb{Z}) \times \mathbb{R}$, which is isotopic to the identity and a fixed point $P$ of $f$.

Definition of the Amount of Rotation of $f$ About a Fixed Point $P$. Let $\tau_{P}$ denote the tangent space to $T \times \mathbb{R}$ at $P$. Each ray emanating from the origin in $\tau_{P}$ intersects the unit circle in $\tau_{P}$ in exactly one point. Hence, we may identify the set $R_{P}$ of such rays with the unit circle, and provide $R_{P}$ with the topology which makes this identification a homeomorphism. The derivative $d f_{P}: \tau_{P} \rightarrow \tau_{P}$ induces a

* Partially supported by NSF contract No. MSC-82-01604 
homeomorphism of $R_{P}$ onto itself which we continue to denote by the same symbol, i.e.

$$
d f_{P}: R_{P} \rightarrow R_{P} .
$$

Since $f$ is assumed to be isotopic to the identity, it is orientation preserving, and consequently, so is $d f_{P}$. It follows that the Poincare rotation number of $d f_{P}: R_{P} \rightarrow R_{P}$ is defined as an element of $T=\mathbb{R} / \mathbb{Z}$.

The amount of rotation $\varrho$ of $f$ about $P$ will be defined to be a real number which is congruent $(\bmod 1)$ to the Poincare rotation number of $d f_{p}$. We further require that $\varrho$ depends continuously on $f$, with respect to the $C^{1}$ topology, for diffeomorphisms which leave $P$ fixed, and that $\varrho=0$ when $f$ is the identity.

It is an easy consequence of known results concerning the topology of the space of diffeomorphisms of a surface that these conditions are consistent and uniquely define $\varrho$, as we will explain in Sect. 4.

Returning to the case of an exact, area-preserving, monotone twist diffeomorphism $f$ and a periodic point $P$ of period $q$, we have the following relation between the amount of rotation $\varrho$ of $f^{q}$ about a given orbit and the Morse index $I$ of $W^{q}$ at the critical point $x$ which corresponds to the orbit:

Theorem 1. If $I$ is even, then $\varrho=I / 2$. If $I$ is odd, then $[I / 2]<\varrho \leqq[I / 2]+1$, with equality if and only if $x$ is a degenerate critical point.

Here, we use the standard notation: $[a]$ denotes the greatest integer $\leqq a$. The definition of $W^{q}$ is given in Sect. 3.

The proof of Theorem 1 is based on a formula of MacKay and Meiss which generalizes a formula of Bountis and Hellemann (see [9, II, formulas (15) and (16)). A similar formula is contained in [2, Appendix H]. Indeed, the formula of MacKay and Meiss implies immediately that $\varrho$ is an integer if and only if $I$ is even or $x$ is a degenerate critical point of $W^{q}$, so that all that is left for us to do is determine which integer it is when $I$ is even or $x$ is a degenerate critical point of $W^{q}$, and which integers it lies between otherwise.

Our second result concerns any orbit, not necessarily periodic. Using a framing of the tangent bundle of $T \times \mathbb{R}$, homotopic to the standard framing, we may still define the amount of rotation $\varrho_{q}(P)$ of $f^{q}$ about a point $P$ in the annulus $T \times \mathbb{R}$. While this has an invariant meaning when $P$ is a periodic point of period $q$, it has no invariant meaning in general, since it depends on the framing of the tangent bundle of $T \times \mathbb{R}$. Nonetheless, it will still be interesting to consider this quantity, because $\lim _{q \rightarrow \infty} q^{-1} \varrho_{q}(P)$ has an invariant meaning, when it exists and the orbit through $P$ is relatively compact (see Sect. 4).

Definition of $\varrho_{q}(P)$. We consider a fixed framing of the tangent bundle of $T \times \mathbb{R}$, homotopic to the standard framing. [By the standard framing, we mean the one which corresponds to the product decomposition $\tau(T \times \mathbb{R})=\tau(T) \times T(\mathbb{R})$ of the tangent bundle.] Given such a framing, we may think of $d f_{P}^{q}$ as a linear mapping of $\mathbb{R}^{2}$ onto itself. Hence, we have an induced mapping

$$
d f_{P}^{q}: R \rightarrow R
$$

on the space of rays emanating from the origin, just as before. We require that $\varrho_{q}(P)$ be congruent $(\bmod 1)$ to the Poincare rotation number of $d f_{P}^{q}: R \rightarrow R$. We further 
require that $\varrho_{q}(P)$ depends continuously on $f$, and that $\varrho_{q}(P)=0$, when $f$ is the identity.

In Sect. 4, we will show that these conditions are consistent and uniquely define $\varrho_{q}(P)$. We will also show that $\limsup _{q \rightarrow \infty} q^{-1} \varrho_{q}(P)$ and $\liminf _{q \rightarrow \infty} q^{-1} \varrho_{q}(P)$ are independent of the framing of the tangent bundle, as long as the orbit through $P$ lies in a compact set.

Definition of $I_{q}(P)$. Orbits of $f$ are in one-one correspondence with equilibrium states (see Sect. 3$)$. Let $x=\left(\ldots, x_{i}, \ldots\right)$ denote the equilibrium state corresponding to the orbit through $P$. By definition of equilibrium state, $\left(x_{0}, \ldots, x_{q}\right)$ is a critical point of an "energy" functional $W_{0 q}$ defined on the space $\mathscr{X}_{[0, q]}\left(x_{0}, x_{q}\right]$ of truncated states $\left(x_{0}^{\prime}, \ldots, x_{q}^{\prime}\right)$ satisfying the boundary condition $x_{0}^{\prime}=x_{0}, x_{q}^{\prime}=x_{q}$ (see the end of Sect. 3). Let $I_{q}(P)$ denote the Morse index of $W_{0 q}$ on $\mathscr{X}_{[0, q]}\left(x_{0}, x_{q}\right)$ at $\left(x_{0}, \ldots, x_{q}\right)$.

Our second result is the following:

Theorem 2. For an exact, area-preserving, monotone twist diffeomorphism, there is a framing of the tangent bundle, which is homotopic to the standard framing, such that

$$
\left[I_{q}(P) / 2\right] \leqq \varrho_{q}(P) \leqq\left[I_{q}(P) / 2\right]+1
$$

holds for all $P$ in the domain of $f$ and all $q \geqq 1$.

The precise framing for which these inequalities are valid is defined at the end of Sect. 4.

Theorems 1 and 2 are easy consequences of appropriate results in linear algebra, stated in Sect. 5 as Theorems 3 and 4, respectively. Theorem 3 is proved in Sect. 6. Theorem 4 follows easily from Theorem 3 (see Sect. 5). We prove that Theorem 3 implies Theorem 1 in Sect. 7 and that Theorem 4 implies Theorem 2 in Sect. 8.

We have written this paper so that the results about linear algebra (Sects. 5 and 6) can be read independently of the rest of this paper.

We conclude this introduction by discussing some examples.

Example 1. Suppose $P$ is a periodic point of period $q$. Clearly, $\varrho_{q l}(P)=\varrho_{q}(P)$, so we obtain from either Theorem 1 or Theorem 2 that

$$
\varrho_{q}(P)=(q / 2) \lim _{N \rightarrow \infty} N^{-1} I_{N}(\xi) .
$$

Note that $d f^{q}(P)$ has a positive eigenvalue if and only if $\varrho_{q}(P) \in \mathbb{Z}$; it has a negative eigenvalue if and only if $\varrho_{q}(P) \equiv \frac{1}{2}(\bmod 1)$; and $d f^{q}(P)$ has an imaginary eigenvalue if and only if $\varrho_{q}(P) \notin 2^{-1} \mathbb{Z}$. In the last case $\lambda=\exp \left(2 \pi i \varrho_{q}(P)\right)$ is an eigenvalue of $d f^{q}(P)$.

Thus, the asymptotic Morse index $\lim _{N \rightarrow \infty} N^{-1} I_{N}(P)$ determines whether $d f^{q}(P)$ has a positive eigenvalue, a negative eigenvalue, or an imaginary eigenvalue, and in the last case determines what the eigenvalues are.

Example 2. Consider an invariant circle $\Gamma$ in the annulus and let $P \in \Gamma$. Suppose that $\Gamma$ is not null-homotopic, so it goes once around the annulus. 
A theorem of G. D. Birkhoff asserts that $\Gamma$ is the graph of a Lipschitz function $T \rightarrow \mathbb{R}$ (where the annulus $=T \times \mathbb{R}$ ). (See [4, Sect. 44], [5, Sect. 3], [6, VIII, Sect. 5], $[10$, Chap. 1], [8], and [18, Sect. 7] for various discussions of this result.) It follows that $\varrho_{q}(P)<1 / 2$, for all $q \geqq 1$. From Theorem 2 , it then follows that $I_{q}(P) \leqq 1$, for all $q \geqq 1$.

Aubry and Le Daeron have proved a stronger result [3]. Under the hypothesis that $\Gamma$ is not null-homotopic and $P \in \Gamma$, they show that if $x=\left(\ldots, x_{i}, \ldots\right)$ is the equilibrium state corresponding to the orbit through $P$, then $\left(x_{0}, \ldots, x_{q}\right)$ is a strict local minimum of $W_{0 q}$ on $\mathscr{X}_{[0, q]}\left(x_{0}, x_{q}\right)$. This beautiful theorem obviously implies that $I_{q}(P)=0$.

Example 3. Again, consider an invariant circle $\Gamma$ in the annulus and let $P \in \Gamma$. This time suppose that $\Gamma$ is homotopically trivial, i.e. it bounds a disk. Under suitable regularity hypothesis on $\Gamma$, it follows from Theorem 2 that the Poincare rotation number $\sigma_{\Gamma}$ of $f \mid \Gamma$ equals $(1 / 2) \lim _{N \rightarrow \infty} N^{-1} I_{N}(P)$. For instance, this holds when $\Gamma$ is $C^{1}$. For, it is easy to see that

so the equation

$$
\sigma_{\Gamma}=\lim _{N \rightarrow \infty} N^{-1} \varrho_{N}(P),
$$

$$
\sigma_{\Gamma}=\frac{1}{2} \lim _{N \rightarrow \infty} N^{-1} I_{N}(P)
$$

then follows immediately from Theorem 2 .

More generally, these assertions hold when $\Gamma$ is locally Lipschitz, i.e., it may be made linear in a neighborhood of a given point by a coordinate change which is Lipschitz and whose inverse is Lipschitz.

\section{Monotone Twist Diffeomorphisms (Definition)}

Throughout this paper, $A$ will denote the cylinder, or annulus, $T \times \mathbb{R}$, and $f$ a $C^{1}$ diffeomorphism of $A$ onto itself, which will be fixed throughout. We will assume that $f$ is orientation preserving and fixes each topological end of $A$. In addition, we assume that $f$ satisfies the following monotone twist condition:

$$
\frac{\partial\left(\pi_{1} f(x, y)\right)}{\partial y}>0,
$$

everywhere, where $\pi_{1}$ is the projection of $T \times \mathbb{R}$ on its first factor.

We will assume that $f$ is area preserving, in the sense that it preserves an area form $\mu=u(x, y) d x d y$, where $u$ is a positive $C^{1}$ function on $A$, and $x(\bmod 1)$ and $y$ are the standard coordinates on $T$ and $\mathbb{R}$. In addition, we will suppose that $f$ is exact, i.e. the flux of $f$ (defined below) vanishes.

It is easy to see that there is a $C^{1}$ embedding $e: A \rightarrow A$ which commutes with the projection of $A=T \times \mathbb{R}$ on its first factor, which is orientation preserving, and which satisfies $e_{*} \mu=d x d y$. In fact, if we further require that $e$ is the identity on the circle $y=0$, then $e$ is uniquely defined by the formulas

$$
\pi_{1} e(x, y)=x, \quad \pi_{2} e(x, y)=\int_{0}^{y} u(x, y) d y,
$$

where $\pi_{i}$ denotes the projection of $A=T \times \mathbb{R}$ on its $i^{\text {th }}$ factor. 
Let $F=e f e^{-1}: e(A) \rightarrow e(A)$. Obviously, $F$ satisfies the monotone twist condition

$$
\frac{\partial\left(\pi_{1}(F(x, y))\right.}{\partial y}>0 \text {. }
$$

Moreover, $F$ preserves the area form $d x d y$. Consequently, $d\left(F^{*}(y d x)-y d x\right)=0$ and the flux $\int_{\gamma}\left(F^{*}(y d x)-y d x\right)$ is independent of $\gamma$, as long as $\gamma$ is a closed curve which winds once around $T \times \mathbb{R}$.

Definition of the Domain $B$ of the Generating Function $h$. Let $\tilde{A}=\mathbb{R}^{2}$ denote the universal covering space of $A$. Let $\tilde{e}: \tilde{A} \rightarrow \tilde{A}$ and $\tilde{F}: \tilde{e}(\tilde{A}) \rightarrow \tilde{e}(\tilde{A})$ be liftings of $e$ and $F$. We set

$$
\begin{aligned}
B= & \left\{\left(x, x^{\prime}\right) \in \mathbb{R}^{2}: \text { there exists } y \in \mathbb{R}\right. \\
& \text { satisfying } \left.(x, y) \in \tilde{e}(\tilde{A}) \text { and } \pi_{1} \tilde{F}(x, y)=x^{\prime}\right\},
\end{aligned}
$$

where $\pi_{1}$ denotes the projection of $\mathbb{R}^{2}$ on its first factor.

The Generating Function $h$. We have assumed that $f$ is exact, i.e. the flux of $f$ vanishes. Consequently, there exists a $C^{2}$ function $H$ on $e(A)$ such that $f^{*}(y d x)$ $-y d x=d H$. Let $\phi: \tilde{e}(\tilde{A}) \rightarrow B$ be defined by $\phi(x, y)=\left(x, \pi_{1} \tilde{F}(x, y)\right)$. The monotone twist condition on $F$ implies that $\phi$ is a diffeomorphism. Let $p r: \tilde{e}(\tilde{A}) \rightarrow e(A)$ denote the projection. We set

$$
h=H \circ p r \circ \phi^{-1} \text {. }
$$

The function $h: B \rightarrow \mathbb{R}$ is what is called in classical mechanics a generating function for $f$ (or $F$ ). It satisfies

$$
F(x, y)=\left(x^{\prime}, y^{\prime}\right) \Leftrightarrow\left\{\begin{array}{l}
y=-\partial h\left(x, x^{\prime}\right) / \partial x, \\
y^{\prime}=\partial h\left(x, x^{\prime}\right) / \partial x^{\prime}
\end{array}\right.
$$

Moreover, $h$ is uniquely defined up to an additive constant by this condition.

\section{Variational Formulation}

An area preserving mapping is an example of a "Hamiltonian system". At least for exact, area preserving, monotone twist mappings, there is a corresponding "Lagrangian formulation". For the Lagrangian formulation, we need the notion of "state". A state will be a bi-infinite sequence of real numbers $\left(\ldots, x_{i}, \ldots\right)$ such that $\left(x_{i}, x_{i+1}\right) \in B$. Let $h_{1}\left(x, x^{\prime}\right)=\partial h\left(x, x^{\prime}\right) / \partial x$ and $h_{2}\left(x, x^{\prime}\right)=\partial h\left(x, x^{\prime}\right) / \partial x^{\prime}$, where $h$ is the generating function for $f$, defined in the previous section. A state $x=\left(\ldots, x_{i}, \ldots\right)$ will be said to be an equilibrium state if

for all $i \in \mathbb{Z}$.

$$
h_{2}\left(x_{i-1}, x_{i}\right)+h_{1}\left(x_{i}, x_{i+1}\right)=0,
$$

In the Lagrangian formulation, one studies states; in the Hamiltonian formulation, one studies orbits of $\tilde{F}$. This amounts to the same thing: there is a oneone correspondence between equilibrium states and orbits of $\widetilde{F}$, defined as follows: Let $x=\left(\ldots, x_{i}, \ldots\right)$ be an equilibrium state. Let $y_{i}=-h_{1}\left(x_{i}, x_{i+1}\right)=h_{2}\left(x_{i-1}, x_{i}\right)$. Since $h$ is a generating function for $f$, i.e. it satisfies (1), we see that $\tilde{F}\left(x_{i}, y_{i}\right)$ $=\left(x_{i+1}, y_{i+1}\right)$. In other words, $\mathcal{O}_{x}=\left(\ldots,\left(x_{i}, y_{i}\right), \ldots\right)$ is an orbit. It follows easily 
from (1) that the correspondence $x \mapsto \mathcal{O}_{x}$ is a bijection of the set of equilibrium states onto the set of orbits of $\tilde{F}$.

Orbits of Type $(p, q)$. Periodic orbits of $F$ do not lift in general to periodic orbits of $\widetilde{F}$. Instead, the lifting $\left(\ldots,\left(x_{i}, y_{i}\right), \ldots\right)$ to $\tilde{e}(\tilde{A})$ of an orbit of $F$ period $q$ satisfies $x_{i+q}=x_{i}+p, y_{i+q}=y_{i}$, for some integer $p$. Such an orbit of $\tilde{F}$ will be said to be of type $(p, q)$.

The corresponding equilibrium state obviously satisfies $x_{i+q}=x_{i}+p$.

States of Type $(p, q)$. We let $\mathscr{X}_{p q}$ denote the set of all states which satisfy $x_{i+q}=x_{i}+p$; such states will be said to be of type $(p, q)$. Clearly, $\mathscr{X}_{p q}$ is an open subset of $\mathbb{R}^{q}$.

The "Energy" Functional. We let $W^{q}$ be the real valued function on $\mathscr{X}_{p q}$, defined by

$$
W^{q}(x)=\sum_{i=0}^{q-1} h\left(x_{i}, x_{i+1}\right) .
$$

Obviously, a state of type $(p, q)$ is an equilibrium state if and only if it is a critical point of $W^{q}$. Hence, there is a one-one correspondence between critical points of $W^{q}$ and orbits of $\widetilde{F}$ of type $(p, q)$.

Let $x$ be a critical point of $W^{q}$. By the Morse index of $W^{q}$ at $x$ (or the Morse index of $x$ ), one means the number of negative eigenvalues of the Hessian matrix (of second partial derivatives) of $W^{q}$ at $x$. By the nullity of $W^{q}$ at $x$ (or the nullity of $x$ ), one means the number of zero eigenvalues of the Hessian matrix of $W^{q}$ at $x$.

The "Energy" Functional on Truncated States. For $m<n$, and $a, b \in \mathbb{R}$, we let $\mathscr{X}_{[m, n]}(a, b)$ denote the set of sequences $\left(x_{m}, \ldots, x_{n}\right)$ such that $\left(x_{i}, x_{i+1}\right) \in B$ [so that $\left(x_{m}, \ldots, x_{n}\right)$ is a "truncated state"] and $x_{m}=a, x_{n}=b$. We let $W=W_{m n}: \mathscr{X}_{[m, n]}(a, b) \rightarrow \mathbb{R}$ be defined by

$$
W(x)=\sum_{i=m}^{n-1} h\left(x_{i}, x_{i+1}\right) .
$$

If $P \in e(A)$ and $x=\left(\ldots, x_{i}, \ldots\right)$ is the equilibrium state associated to $P$, we let $I_{q}(P)$ be the Morse index at $\left(x_{0}, \ldots, x_{q}\right)$ of $W_{0 q}: \mathscr{X}_{[0, q]}\left(x_{0}, x_{q}\right) \rightarrow \mathbb{R}$.

\section{Amount of Rotation}

Let $g$ be a $C^{1}$ diffeomorphism of $A$, isotopic to the identity, and let $P$ be a fixed point of $g$. In the introduction, we defined the amount $\varrho$ of rotation of $g$ about $P$. To show that this is a correct definition, we must show that there is an isotopy connecting $g$ to the identity and leaving $P$ fixed, and that $\varrho$ is independent of the isotopy chosen.

Let $\mathscr{D}$ denote the space of $C^{1}$ diffeomorphisms of $A$ with the $C^{1}$ topology. Let $P \in A$. We see easily that the evaluation mapping $e v_{p}: \mathscr{D} \rightarrow A$, defined $e v_{p}(g)=g(P)$, is a locally trivial fibration. Let $\mathscr{D}_{0}$ denote the connected component of the identity in $\mathscr{D}$. It is easy to see that $\mathscr{D}_{0}$ is locally arcwise connected. Consequently, $\mathscr{D}_{0}$ is the set of diffeomorphisms of $A$ which are isotopic to the identity. It is also well known to differential topologists that $e v_{p}: \mathscr{D}_{0} \rightarrow A$ is a homotopy equivalence. This fact is 
an easy consequence of the theorem proved by Smale in [20]. (For another proof of Smale's theorem, see [7].) Let $\mathscr{D}_{0 P}=e v_{p}^{-1}(P) \cap \mathscr{D}_{0}$. From the above comments, it follows that $\mathscr{D}_{0 P}$ is a contractible space.

It follows that for any $g \in \mathscr{D}_{0 P}$, there is an isotopy to the identity which leaves $P$ fixed. Moreover, two such isotopies are homotopic (rel. endpoints) as curves in $\mathscr{D}_{0 P}$. The fact that $\varrho$ is well defined follows immediately.

The proof that the definition of $\varrho_{q}(P)$ is consistent is similar. Again two isotopies of $g$ with the identity give the same value of $\varrho_{q}(P)$, provided that they are homotopic (rel. endpoints) as curves in $\mathscr{D}_{0}$. However, since $\mathscr{D}_{0}$ is homotopy equivalent to $A$ and therefore to $T$, it is not true that two such isotopies are homotopic (rel. endpoints). But it is true that for any two isotopies $I_{1}, I_{2}$ of $A$ connecting $g$ to the identity, there is a 1-parameter family $R$ of rotations of $A$, beginning and ending at the identity, such that $I_{1}$ is homotopic (rel. endpoints) to the juxtaposition $R * I_{2}$ in $\mathscr{D}_{0}$. Juxtaposing with $R$ does not change $\varrho_{q}(P)$, since the given framing of the tangent bundle is homotopic to the standard one, so we obtain that the definition of $\varrho_{q}(P)$ is consistent.

Independence from the Framing. Let $t$ and $t^{\prime}$ be two framings of the tangent bundle of $T \times \mathbb{R}$, both homotopic to the identity. Let $K$ be a compact set in $T \times \mathbb{R}$. It is easy to see that there is a constant $C>0$ such that if $P$ and $f^{q} P$ are in $K$, then

$$
\left|\varrho_{q}^{\prime} P-\varrho_{q} P\right| \leqq C,
$$

where $\varrho_{q} P$ and $\varrho_{q}^{\prime} P$ are the rotation numbers associated to the framings $t$ and $t^{\prime}$, respectively.

It follows immediately that

$$
\liminf _{q \rightarrow \pm \infty} \varrho_{q}(P) / q, \quad \limsup _{q \rightarrow \pm \infty} \varrho_{q}(P) / q,
$$

are independent of the framing chosen, as long as the orbit of $f$ through $P$ lies in a compact subset of $A$.

Linear Algebra. There is an alternative, but more specialized, definition of the amount $\varrho$ of rotation about a periodic point $P$ and of the number $\varrho_{q}(P)$, which will play an important role in the proofs.

Before stating the definition, we need some elementary remarks about linear algebra.

Let $G L(2, \mathbb{R})^{0}$ denote the identity component of $G L(2, \mathbb{R})$, i.e. the set of $2 \times 2$ matrices with real entries and positive determinant. Let $R$ denote the set of rays emanating from the origin in $\mathbb{R}^{2}$ with its usual topology, so that $R$ is homeomorphic to the circle. An element $L$ of $G L(2, \mathbb{R})^{0}$ induces an orientation preserving homeomorphism of $R$. By the rotation number $\varrho(L)$, we will mean the Poincaré rotation number of the induced homeomorphism of $R$. Then $\varrho(L)$ is defined $(\bmod 1)$.

If $L$ has a positive eigenvalue, then $\varrho(L) \equiv 0(\bmod 1)$; if it has a negative eigenvalue, then $\tilde{\varrho}(L) \equiv \frac{1}{2}(\bmod 1)$; if its eigenvalues are imaginary, then $\lambda=(\operatorname{det} L)^{1 / 2} \exp (2 \pi i \varrho(L))$ is one of them.

Set $G=G L(2, \mathbb{R})^{0}$ and let $\widetilde{G}$ be its universal covering group. Let $\varrho$ be the unique continuous, real valued function on $\widetilde{G}$ such that $\varrho(1)=0$ and $\varrho \circ \pi \equiv \varrho(\bmod 1)$, where 
$\pi$ denotes the projection $\widetilde{G} \rightarrow G$. If $L \in \widetilde{G}$, the number $\varrho(\widetilde{L})$ will be called the rotation number of $L$.

Alternative Definition of the Amount of Rotation. Now we give an alternative definition for the amount of rotation about a point for $f$. This is more specialized than our previous definition, since it applies only to monotone twist diffeomorphisms and only for framings of the tangent bundle for which one of the basis vectors points in the vertical, upward direction. We will call such a framing special. This alternative definition is the definition which we will use in the proof of Theorems 1 and 2. When it applies, it is equivalent to our previous definition.

We suppose given a special framing of the tangent bundle of $T \times \mathbb{R}$. For $P \in e(A)$, the derivative $d F_{P}: \tau_{P} \rightarrow \tau_{F(P)}$ may be thought of as an element of $G$, since $\tau_{P}$ and $\tau_{F(P)}$ are identified with $\mathbb{R}^{2}$. Since $F$ satisfies the monotone twist condition and an upper vertical vector is one of the basis vectors at any point, it follows that $\varrho\left(d F_{P}\right) \in\left[0, \frac{1}{2}\right)(\bmod 1)$.

We let $\tilde{d} f_{P}$ be the unique lift of $d f_{P}$ to $\tilde{G}$ which satisfies $0 \leqq \varrho\left(\widetilde{d} f_{P}\right)<1 / 2$.

If $q>1$, we let $\tilde{d} F_{P}^{q}=\tilde{d}_{F^{q-1}{ }_{P}} F \ldots \tilde{d} F_{P}$. Then $\widetilde{d} F_{P}^{q}$ is a lift to $\widetilde{G}$ of $d F_{P}^{q}$, for all $q \geqq 1$.

It is easy to see that $\varrho_{q}\left(e^{-1} P\right)=\varrho\left(\tilde{d} f_{P}^{q}\right)$, where the left side is the quantity defined in the introduction, and $\varrho: \widetilde{G} \rightarrow \mathbb{R}$ is the rotation number defined earlier in this section. Indeed, we could have defined $\widetilde{d} F_{P}^{q}$ by requiring it to be a lift of $d F_{P}^{q}$, continuous in $F$, and equal to 1 when $F=i d$. Of course, to have the formula $\varrho_{q}\left(e^{-1} P\right)=\varrho\left(\tilde{d} F_{P}^{q}\right)$, we must use the same framing for both sides, and it must be special in order for the right side to be defined.

The Framing. The inequalities which appear in Theorem 2 depend on a particular framing of the tangent bundle of $A$. This framing may be defined as follows: We may pull back the standard framing of $\mathbb{R}^{2}$ to a framing of $A$ via $\phi \circ \tilde{e}$, since $\phi \circ \tilde{e}: \widetilde{A} \rightarrow \mathbb{R}^{2}$ is a diffeomorphism of $\widetilde{A}$ onto the open subset $B$ of $\mathbb{R}^{2}$. Let $(x, y) \in \tilde{A}$. We have $\phi \tilde{e}(x, y)=\left(x, x^{\prime}\right)$, for a suitable $x^{\prime} \in \mathbb{R}$. Moreover, $\phi \tilde{e}(x+1, y)$ $=\left(x+1, x^{\prime}+1\right)$. Consequently, the framing of the tangent bundle of $A$ is invariant under the Deck transformations of $\tilde{A}$ over $A$, and so it can be pushed down to a framing $t$ of the tangent bundle of $A$. It is easily seen that $t$ is homotopic to the standard framing of $A$.

We will show in Sect. 8 that the inequalities of Theorem 2 are valid for the framing $t$ of the tangent bundle of $A$.

\section{The Mappings $\Phi$ and $\Psi$}

We let $\Sigma_{q}$ denote the set of sequences $\alpha=\left(a_{1}, \ldots, a_{q}, b_{1}, \ldots, b_{q}\right)$ of real numbers such that $b_{i}<0$, for all $i$. To any such sequence, we associate certain matrices, $\Psi(\alpha)$, $\bar{\Phi}_{1}(\alpha), \ldots, \bar{\Phi}_{q}(\alpha), \bar{\Phi}(\alpha)$, as follows.

We let $\Psi(\alpha)$ be the $q \times q$ symmetric matrix $\left[c_{i j}\right]$ with real entries, defined as follows. If $q=1$, we let $c_{11}=a_{1}+2 b_{1}$. If $q=2$, we let

$$
\left(\begin{array}{ll}
c_{11} & c_{12} \\
c_{21} & c_{22}
\end{array}\right)=\left(\begin{array}{cc}
a_{1} & b_{1}+b_{2} \\
b_{1}+b_{2} & a_{2}
\end{array}\right) .
$$

If $q \geqq 3$, we let $c_{i i}=a_{i}, c_{i, i+1}=c_{i+1, i}=b_{i}, c_{q 1}=c_{1 q}=b_{q}$, and $c_{i j}=0$, otherwise. 
For $1 \leqq i \leqq q$, we set

$$
\bar{\Phi}_{i}(\alpha)=\left(\begin{array}{cc}
0 & 1 \\
-\frac{b_{i-1}}{b_{i}} & -\frac{a_{i}}{b_{i}}
\end{array}\right),
$$

where we set $b_{0}=b_{q}$. We set $\bar{\Phi}(\alpha)=\bar{\Phi}_{q}(\alpha) \ldots \bar{\Phi}_{1}(\alpha)$. It is clear that $\bar{\Phi}_{i}(\alpha) \in G$ and

$$
\bar{\varrho}\left(\bar{\Phi}_{i}(\alpha)\right) \in\left[0, \frac{1}{2}\right)(\bmod 1), \quad i=1, \ldots, q .
$$

We let $\Phi_{i}(\alpha)$ be the unique lift of $\bar{\Phi}_{i}(\alpha)$ to $\widetilde{G}$ such that

$$
0 \leqq \varrho\left(\Phi_{i}(\alpha)\right)<\frac{1}{2}, \quad i=1, \ldots, q .
$$

We let

$$
\Phi(\alpha)=\Phi_{q}(\alpha) \ldots \Phi_{1}(\alpha)
$$

It is clear that $\Phi(\alpha)$ is a lift of $\Phi(\alpha)$ to $\tilde{G}$.

Here is the result in linear algebra which leads to the other results in this paper:

Theorem 3. Let $\alpha \in \Sigma_{q}$. Let $I=I(\alpha)$ be the number of negative eigenvalues of $\Psi(\alpha)$ (with multiplicities counted), and let $Z=Z(\alpha)$ be the multiplicity of zero as an eigenvalue of $\Psi(\alpha)$. If I is even, then $Z \leqq 1$ and $\varrho \Phi(\alpha)=I / 2$. If $I$ is odd, then $Z \leqq 2$, and $[I / 2]<\varrho \Phi(\alpha) \leqq[I / 2]+1$, with equality if and only if $Z \geqq 1$.

By the definition of $\Phi(\alpha), \operatorname{det} \bar{\Phi}(\alpha)=1$. Consequently, $\varrho \Phi(\alpha)$ is an integer if and only if trace $\bar{\Phi}(\alpha) \geqq 2$. We have the following formula for trace $\bar{\Phi}(\alpha)$, due to R. MacKay and J. Meiss, which generalizes a formula due to J. Greene. D. Goroff has pointed out to me that similar formulas have been obtained by Aubry et al. [2, Appendix H]:

Lemma 1. $\operatorname{trace} \bar{\Phi}(\alpha)-2=\operatorname{det} \Psi(\alpha) / \prod_{i=1}^{q}\left(-b_{i}\right)$.

Note that Lemma 1 immediately implies this much of Theorem $3: \varrho \Phi(\alpha)$ is an integer if and only if $I$ is even or $Z \geqq 1$. All that is left to do to prove Theorem 3 is to determine which integer it is if $I$ is even or $Z \geqq 1$, and which integers it lies between otherwise. This will be done in Sect. 6 .

Let $\Psi^{\prime}(\alpha)$ be the $(q-1) \times(q-1)$ symmetric matrix obtained by omitting the last row and last column in $\Psi(\alpha)$. The following is an easy consequence of Theorem 3 .

Theorem 4. Let $\alpha \in \Sigma_{q}$. Let $I^{\prime}=I^{\prime}(\alpha)$ be the number of negative eigenvalues of $\Psi^{\prime}(\alpha)$ (with multiplicities counted). Then

$$
\left[I^{\prime} / 2\right] \leqq \varrho \Phi(\alpha) \leqq\left[I^{\prime} / 2\right]+1 .
$$

Proof. In view of the definitions of $\Psi(\alpha)$ and $\Psi\left(\alpha^{\prime}\right)$, we have either $I=I^{\prime}$ or $I=I^{\prime}+1$. There are four cases, according to whether $I^{\prime}$ is odd or even and whether $I=I^{\prime}$ or $I=I^{\prime}+1$. The proof of the theorem then reduces to checking these four cases, and each of them follows trivially from Theorem 3 . 


\section{Proof of Theorem 3}

Let $\Sigma_{q}^{0}$ denote the set of $\alpha \in \Sigma_{q}$ for which $\operatorname{det} \Psi(\alpha) \neq 0$. We provide $\Sigma_{q}^{0}$ with the topology induced from the standard topology on $\mathbb{R}^{2 q}$.

It is obvious that there exists $C_{0}>0$ (depending only on $q$ ) such that if $\alpha=\left(a_{1}, \ldots, a_{q},-1, \ldots,-1\right)$ and $\left|a_{i}\right| \geqq C_{0}$, for each $i$, then $\alpha \in \Sigma_{q}^{0}$.

Lemma 2. Let $C \geqq C_{0}$. Each connected component of $\Sigma_{q}^{0}$ contains an element $\alpha=\left(a_{1}, \ldots, a_{q},-1, \ldots,-1\right)$ such that $\left|a_{i}\right|=C$ for $i=1, \ldots, q$.

Proof. Consider $\alpha=\left(a_{1}, \ldots, a_{q}, b_{1}, \ldots, b_{q}\right) \in \Sigma_{q}^{0}$. First, consider the case when $C \geqq\left|a_{i}\right|$, for all $i$. Choose one $i$ satisfying $1 \leqq i \leqq q$ and let $\alpha_{t}=\left(a_{1}, \ldots, a_{i-1}, a_{i}+t a_{i}^{*}, a_{i+1}, \ldots, a_{q}, b_{1}, \ldots, b_{q}\right)$, for some real number $a_{i}^{*}$. Then

$$
\operatorname{det} \Psi\left(\alpha_{t}\right)=\Delta+t \Delta^{\prime} a_{i}^{*},
$$

where $\Delta$ is the determinant of $\Psi(\alpha)$ and $\Delta^{\prime}$ is the $(q-1) \times(q-1)$ minor determinant of $\Delta$ obtained by deleting the $i^{\text {th }}$ row and $i^{\text {th }}$ column. If we choose $a_{i}^{*}$ so that $\Delta^{\prime} a_{i}^{*}$ vanishes or has the same sign as $\Delta$, then $\alpha_{t} \in \Sigma_{q}^{0}$ for all $t \geqq 0$. Consequently, we may deform $\alpha$ in $\Sigma_{q}^{0}$ to make $\left|a_{i}\right|=C$, without changing the $b_{i}$ 's or $a_{j}$ 's for $j \neq i$. Since we may do this for each $i$, there is $\alpha^{\prime}=\left(a_{1}^{\prime}, \ldots, a_{q}^{\prime}, b_{1}, \ldots, b_{q}\right)$ in the same connected component of $\Sigma_{q}^{0}$ as $\alpha$, for which $\left|a_{i}^{\prime}\right|=C$ for $1 \leqq i \leqq q$.

If $C$ is large enough (in relation to $\alpha$ ), the above argument applies. Moreover, we may deform $\alpha^{\prime}$ to $\alpha^{\prime \prime}=\left(a_{1}^{\prime}, \ldots, a_{q}^{\prime},-1, \ldots,-1\right)$ in $\Sigma_{q}^{0}$. This shows that for $C$ large enough, we have the conclusion of Lemma 2. By the definition of $C_{0}$, this conclusion then follows for all $C \geqq C_{0}$.

Proof of Theorem 3. By Lemma 1, it is enough to prove Theorem 3 for a single element of a given component of $\Sigma_{q}^{0}$ in order to prove it for all elements in that component. By Lemma 2, if $C \geqq C_{0}$, each component of $\Sigma_{q}^{0}$ contains an element $\alpha=\left(a_{1}, \ldots, a_{q},-1, \ldots,-1\right)$ for which $\left|a_{i}\right|=C$. For such $\alpha$, we have

$$
\bar{\Phi}_{i}(\alpha)=\left(\begin{array}{rr}
0 & 1 \\
-1 & \pm C
\end{array}\right) .
$$

It follows easily that for $C$ large enough $\varrho \Phi(\alpha)$ is very near to $\frac{1}{2} \times$ (the number of negative diagonal entries of $\Psi(\alpha)$ ). Clearly, if $C$ is large enough, then the number of negative diagonal entries of $\Psi(\alpha)$ equals $I(\alpha)$. Therefore, $\varrho \Phi(\alpha)$ is very near to $I(\alpha) / 2$. We then obtain Theorem 3 when $I$ is odd. By Lemma $1, \varrho \Phi(\alpha)$ is an integer if $I$ is even, so we obtain Theorem 3 for $\operatorname{such} \alpha$, too.

It remains to consider the case $\alpha=\left(a_{1}, \ldots, a_{q}, b_{1}, \ldots, b_{q}\right) \in \Sigma_{q} \backslash \Sigma_{q}^{0}$, i.e. the case $Z \geqq 1$. We will say that a minor of $\Psi(\alpha)$ is symmetric if the set of rows which occurs in that minor is the same as the set of columns which occurs. We let $r$ be the size of a largest symmetric minor of $\Psi(\alpha)$ with non-vanishing determinant and let $\Delta^{\prime}$ be the corresponding determinant. We let $i_{1}<\ldots<i_{q-r}$ be the indices of the rows (or columns) which are deleted in order to obtain this minor. For $t=\left(t_{1}, \ldots, t_{q-r}\right)$, we let $\alpha_{t}=\left(a_{1 t}, \ldots, a_{q t}, b_{1}, \ldots, b_{q}\right)$, where $a_{j t}=a_{j}$ if $j \notin\left\{i_{j}, \ldots, i_{q-r}\right\}$ and $a_{j t}=a_{j}+t_{k}$ if $j=i_{k}$. For $0 \leqq k \leqq q-r$, let $\Psi\left(\alpha_{t}\right)_{k}$ denote the $(r+k) \times(r+k)$ minor of $\Psi\left(\alpha_{t}\right)$ obtained by deleting rows and columns $i_{k+1}, \ldots, i_{q-r}$. We let $\Delta_{t k}$ denote the determinant of $\Psi\left(\alpha_{t}\right)_{k}$. From the fact that $\Psi(\alpha)_{0}$ is a largest symmetric minor of $\Psi(\alpha)$ with non- 
vanishing determinant, it follows easily that

$$
\Delta_{t k}=t_{1} \ldots t_{k} \Delta^{\prime} \text {. }
$$

For $t_{1} \neq 0, \ldots, t_{q-r} \neq 0$, we have that $\Psi\left(\alpha_{t}\right)_{k}$ is non-singular for $k=0, \ldots, q-r$, and

$$
\begin{aligned}
& \text { index } \Psi\left(\alpha_{t}\right)_{k}-\operatorname{index} \Psi\left(\alpha_{t}\right)_{k-1}=1, \quad \text { if } t_{k}<0 \text {, } \\
& =0, \text { if } t_{k}>0 \text {. }
\end{aligned}
$$

For, the parity of the index of $\Psi\left(\alpha_{t}\right)_{k}$ is determined by the sign of $\Delta_{t k}$ and the difference of the above two indices is clearly either 0 or 1 .

These observations and the fact that $I=\operatorname{index} \Psi(\alpha)=\operatorname{index} \Psi(\alpha)_{0}$ have the following consequence: Let $I \leqq I^{*} \leqq I+q-r$. If we choose $I^{*}-I$ of the $t_{i}$ 's to be negative and the remaining ones to be positive, then the index of $\Psi\left(\alpha_{t}\right)=I^{*}$. On the other hand,

$$
\lim _{\lambda \rightarrow 0} \varrho \Phi\left(\alpha_{\lambda t}\right)=\varrho \Phi(\alpha) \text {. }
$$

In particular, if $I^{*}$ is an even integer in $[I, I+q-r]$, then we may choose $I^{*}-I$ of the $t_{i}$ 's to be negative. For all $\lambda>0$, we have $\alpha_{\lambda t} \in \Sigma_{q}^{0}$ and the index of $\Psi\left(\alpha_{\lambda t}\right)$ is $I^{*}$. Since $\alpha_{t} \in \Sigma_{q}^{0}$, Theorem 3 applies to it, and we have $\varrho \Phi\left(\alpha_{\lambda t}\right)=I^{*} / 2$. Then,

$$
\varrho \Phi(\alpha)=\lim _{\lambda \rightarrow 0} \varrho \Phi\left(\alpha_{\lambda t}\right)=I^{*} / 2 .
$$

It follows that $[I, I+q-r]$ contains at most one even integer, namely, $2 \varrho \Phi(\alpha)$. Hence, $q-r \leqq 1$ if $I$ is even and $q-r \leqq 2$ if $I$ is odd. Consequently, $Z \leqq 1$ if $I$ is even and $Z \leqq 2$ if $I$ is odd. The other conclusions of Theorem 3 can be obtained from (2), the fact that they have already been proved for $\alpha_{t} \in \Sigma_{q}^{0}$, and Lemma 1 .

\section{Proof that Theorem 3 Implies Theorem 1}

Let $P$ be a periodic point of type $(p, q)$ and $x=\left(\ldots, x_{i}, \ldots\right) \in \mathscr{X}_{p q}$ the corresponding state. By definition, the quantity $I$ which appears in Theorem 1 is the number of negative eigenvalues of the Hessian matrix of $W^{q}: \mathscr{X}_{p q} \rightarrow \mathbb{R}$ at $x$. This Hessian matrix is $\Psi(\alpha)$, where $\alpha=\left[a_{1}, \ldots, a_{q}, b_{1}, \ldots, b_{q}\right]$,

$$
\begin{gathered}
a_{i}=\left(\partial^{2} W / \partial x_{i}^{2}\right)(x)=h_{11}\left(x_{i}, x_{i+1}\right)+h_{22}\left(x_{i-1}, x_{i}\right), \\
b_{i}=\left(\partial^{2} W / \partial x_{i} \partial x_{i+1}\right)(x)=h_{12}\left(x_{i}, x_{i+1}\right) .
\end{gathered}
$$

If we choose a framing of the tangent bundle of $T \times \mathbb{R}$, then $d f_{Q}$ may be thought of as an isomorphism of $\mathbb{R}^{2}$, and hence as a $2 \times 2$ matrix for any $Q \in T \times \mathbb{R}$. For the framing which we defined at the end of Sect. 4, we have

$$
d f_{f^{i} p}=\bar{\Phi}_{i}(\alpha) \text {. }
$$

For, since $x$ is an equilibrium state, we have

$$
h_{12}\left(x_{i-1}, x_{i}\right) d x_{i-1}+\left(h_{22}\left(x_{i-1}, x_{i}\right)+h_{11}\left(x_{i}, x_{i+1}\right)\right) d x_{i}+h_{12}\left(x_{i}, x_{i+1}\right) d x_{i+1}=0 \text {, }
$$

or

$$
\begin{gathered}
d x_{i}=d x_{i} \\
d x_{i+1}=-\frac{b_{i-1}}{b_{i}} d x_{i-1}-\frac{a_{i}}{b_{i}} d x_{i},
\end{gathered}
$$

from which (3) follows. 
Let $\varrho$ be the quantity which appears in Theorem 1. By the alternative definition of the amount of rotation $\varrho$, described in Sect. 4, it follows that $\varrho=\varrho \Phi(\alpha)$. To see this, compare the definition of $\Phi(\alpha)$ to the definition of $\widetilde{d} F_{P}^{q}$ given in the discussion of the alternative direction of the amount of rotation.

It is then clear that Theorem 1 is a special case of Theorem 3.

\section{Proof that Theorem 4 Implies Theorem 2}

We claim that the inequality $\left[I_{q}(P) / 2\right] \leqq \varrho_{q}(P) \leqq\left[I_{q}(P) / 2\right]+1$ is valid for the framing of the tangent bundle which we defined at the end of Sect. 4 . This is the same framing as we used in the proof of Sect. 7. The proof that Theorem 4 implies Theorem 2 is like the proof that Theorem 3 implies Theorem 1, with appropriate notational changes.

By definition, the quantity $I_{q}(P)$ which appears in Theorem 2 is the number of negative eigenvalues of the Hessian matrix of $W_{0 q}: \mathscr{X}_{[0, q]}\left(x_{0}, x_{q}\right) \rightarrow \mathbb{R}$ at $\left(x_{0}, \ldots, x_{q}\right)$. This Hessian matrix is $\Psi^{\prime}(\alpha)$, where $\alpha$ is as in Sect. 7. Hence $I_{q}(P)=I^{\prime}(\alpha)$. The argument given in Sect. 7 that $\varrho=\varrho \Phi(\alpha)$ now shows $\varrho_{q}(I)=\varrho \Phi(\alpha)$.

Acknowledgement. The idea of this paper was conceived while I was on sabbatical leave at IHES in August of 1983. I would like to thank IHES for its hospitality during the academic year 1982-83.

\section{References}

1. Aubry, S.: The new concept of transitions by breaking of analyticity in a crystallographic model. In: Solitons and condensed matter. Proceedings of the symposium on nonlinear (soliton) structure and dynamics in condensed matter, Oxford, England, June 27-29, 1978, Bishop, A.R., Schneider, T. (eds.). Berlin, Heidelberg, New York: Springer 1978

2. Aubry, S., Le Daeron, P.Y., André, G.: Classical ground-states of a one-dimensional model for incommensurate structures. Preprint (1982)

3. Aubry, S., Le Daeron, P.Y.: The discrete Frenkel-Kontorava model and its extensions. I. Exact results for the ground-states. Physica 8D, 381-422 (1983)

4. Birkhoff, G.D.: Surface transformations and their dynamical applications. Acta Math. 43, 1-119 (1922). Reprinted in Collected Mathematical Papers, Vol. II. New York: Am. Math. Soc. 1950, 111-229

5. Birkhoff, G.D.: Sur quelques courbes fermées remarquables. Bull. Soc. Math. France 60, 1-26 (1932). Reprinted in Collected Mathematical Papers, Vol. II. New York: Am. Math. Soc. 1950, 418-443

6. Birkhoff, G.D.: Dynamical Systems. Am. Math. Soc. Colloquium Publications, Vol. IX: Providence, R.I.: AMS 1927

7. Earle, C.J., Eells, J.: A fibre bundle description of Teichmüller theory. J. Diff. Geom. 3, 19-43 (1969)

8. Fathi, A.: Une interprétation plus topologique de la démonstration du théorème de Birkhoff. Appendix to Chap. 1 of [10]

9. Greene, J.M.: A method for determining a stochastic transition. J. Math. Phys. 20, 1183-1201 (1979)

10. Herman, M.R.: Introduction à l'Etude les courbes invariantes par les diffeomorphismes de l'anneau. Asterisque 103-104 (1983)

11. Katok, A.: Some remarks on the Birkhoff and Mather twist theorems. Ergodic Theor. Dyn. Syst. 2, 183-194 (1982) 
12. Katok, A.: More about Birkhoff periodic orbits and Mather sets for twist maps. Ergodic Theor. Dyn. Syst. (to appear)

13. MacKay, R.S., Meiss, J.D.: Linear stability of periodic orbits in Lagrangian systems. Phys. Lett. 98 A, 92-94 (1984)

14. Mather, J.N.: Existence of quasi-periodic orbits for twist homeomorphisms of the annulus. Topology 21, 457-467 (1982)

15. Mather, J.N.: Concavity of the Lagrangian for quasi-periodic orbits. Comment. Math. Helvetici 57, 356-376 (1982)

16. Mather, J.N.: Non-uniqueness of solutions of Percival's Euler-Lagrange equation. Commun. Math. Phys. 86, 465-476 (1982)

17. Mather, J.N.: Glancing billiards. Ergodic Theor. Dyn. Syst. 2, 397-403 (1982)

18. Mather, J.N.: Non-existence of invariant circles. Ergodic Theor. Dyn. Syst. (to appear)

19. Mather, J.N.: A criterion for the non-existence of invariant circles. Preprint, Aug. 9, 1982

20. Smale, S.: Diffeomorphisms of the 2-sphere. Proc. Am. Math. Soc. 10, 621-626 (1959)

Communicated by A. Jaffe

Received March 2, 1984 
